\title{
Transcriptome comparison of physiological divergence between two ecotypes of Portulaca oleracea
}

\author{
M. WU ${ }^{1,2,+}$, S. FU ${ }^{1,2,3,+}$, W. JIN ${ }^{1,2}$, W.Z. XIANG ${ }^{1,2}$, W.C. ZHANG ${ }^{1,2}$, and L. CHEN ${ }^{1,2,3,{ }^{*}}$ \\ ${ }^{1}$ Hubei Key Laboratory of Edible Wild Plants Conservation \& Utilization, Huangshi 435002, P.R. China \\ ${ }^{2}$ The College of Life Science, Hubei Normal University, Huangshi 435002, P.R. China \\ ${ }^{3}$ Hubei Engineering Research Center of Special Wild Vegetables Breeding and Comprehensive \\ Utilization Technology, Huangshi 435002, P.R.China
}

*Corresponding author: E-mail: chliang666@aliyun.com

\begin{abstract}
According to the survey, wild purslane (Portulaca oleracea L.) has two different ecotypes, one with the red stem living in an arid environment and one with the green stem living in a humid environment. In order to explore the physiological response strategies of plants to environmental changes, these two ecotypes of purslane were selected as experimental materials. Physiological indices were determined and transcriptome analysis was carried out to screen the differentially expressed genes (DEGs) from two ecotypes of purslane. The results showed that the content of soluble sugars, proline, anthocyanins, and chlorophylls was significantly different, and most DEGs belonged to the WRKY and NAC families. Finally, the results of transcriptome analysis were verified by real-time qPCR. Therefore, it can be inferred that the transcription factor (TF) families may play an important role in physiological response strategies by regulating the changes of anthocyanins and osmotic regulators (soluble sugars, proline, etc.) through the abscisic acid signalling pathway. This will accelerate the study of purslane at the molecular level, provide corresponding theoretical support for its artificial domestication, and drought breeding.
\end{abstract}

Keywords: anthocyanin, chlorophyll, differential gene expression, Portulaca oleracea, proline, purslane ecotypes, stress tolerance, sugars.

\section{Introduction}

Drought caused by global climate change and population growth has been one of the main factors restricting plant growth and development. Drought is mainly the lack of water in the soil provided to plants and it can have adverse effects on the metabolism of plants. However, plants can adapt to the drought stress mainly through three adaptive strategies: morphology (reduction of leaf area, leaf shape, wax content, efficient rooting system, stability of yield and branch number), physiology (regulation of transpiration, water use efficiency, stomatal activity, and osmotic adjustment), and biochemical reactions (accumulation of proline, polyamines, trehalose, increase of nitrate reductase activity, and storage of saccharides) (Haworth et al. 2013, Ammar et al. 2014, Conesa et al. 2016). In response to water stress, abscisic acid (ABA) accumulation is one of the key adaptive mechanisms (Pennisi 2017, Phillips and Sussman 2019).

The rapid development of molecular biology in recent years has promoted research on the regulation mechanisms of plant responses to the environment at

Received 19 November 2020, last revision 15 February 2021, accepted 19 February 2021.

Abbreviations: ABA - abscisic acid; DEGs - differentially expressed genes; GO - gene ontology; TF - transcription factor.

Acknowledgements: We thank the Natural Science Foundation of Hubei Province and the Research and Innovation Projects for Postgraduates of Hubei Normal University for the project support. This research was also supported by the Natural Science Foundation of Hubei Province (No. 2018CFC858) and the Research and Innovation Projects for Postgraduates of Hubei Normal University (No. 20200138).

${ }^{+}$These authors contributed equally.

Conflict of interest: The authors declare that they have no conflict of interest. 
the molecular level. Transcription factors (TFs) play key roles in the regulation of plants resistance to adverse conditions. Therefore, it is necessary to understand the specific mechanisms of different TF families in response to drought stress to improve the drought resistance of crop cultivars. More than $30 \mathrm{TFs}$ families, such as the DREB, MYB, NAC, bZIP, and WRKY families, have been identified as being associated with drought stress. For example, several basic WRKY family genes play an important role in adaptation to water stress. AtWRKY57 is crucial in drought stress signalling (Jiang et al. 2016), overexpression of $B d W R K Y 36$ in different transgenic plants improve tolerance to drought (Sun et al. 2015), and coexpression of AtCKX1 and AtWRKY6 result in resistance to drought and heat stresses in tobacco plants (Macková et al. 2013). In general, the differential expression of TF in plants under drought stress will reveal the mechanism of drought stress at the molecular level, which is also critical to the cultivation and breeding of Portulaca oleracea.

Purslane (Portulaca oleracea L.) is eaten raw or cooked and used for feeding pigs and cows. It is rich in $\omega 3$ polyunsaturated fatty acids and antioxidants, which have high medicinal value (Simopoulos 2008, Uddin et al. 2012). In addition, Portulaca oleracea is able to produce enough biomass under moderate salt stress because of its higher salt tolerance than any other vegetable crops (Yazici et al. 2007, Kafi and Rahimi 2011, Alam et al. 2014a,b). These characteristics give purslane a competitive advantage over many other cultivated crops and it might be a promising crop for novel biologically active substances and essential compounds for human nutrition (Alam et al. 2016).

There are many studies on the viability of purslane under adverse circumstances, as well as its nutritional and medicinal value. However, there are few reports on the effect of stress on gene transcription. Wild purslane has two different ecotypes (the red-stem ecotype in arid environment and the green-stem ecotype in humid environment). The physiological characteristics of two different ecotypes of purslane had been compared, and the transcriptome of them had been analyzed in this paper. The present study aims to accelerate the research of Portulaca olerace a a molecular level and so provide the theoretical support for the domestication of purslane and breeding it for enhanced drought tolerance

\section{Materials and methods}

Plant materials and study sites: The research areas were located at the Hubei Normal University, Huangshi City, Hubei Province. The mean annual precipitation was $1386 \mathrm{~mm}$, and the mean annual temperature was $17^{\circ} \mathrm{C}$. Two ecotypes of purslane were selected as the plant materials, which were gathered in the Qionglin Garden (located at the Hubei Normal University). One was the green-stem purslane, which is growing under the moist and shady habitat. The other one was collected from the sunny and dry areas, which was the red-stem purslane (Table 2 Suppl.). Areas within each site with even distribution of purslane were selected; 6 - 10 quadrats $\left(1 \mathrm{~m}^{2}\right)$ about $5-10 \mathrm{~m}$ apart were established as sampling plots.

Physiological characteristics determination: The content of proline, soluble sugars, chlorophylls, and anthocyanins of the two ecotypes of purslane were measured and the ecotypes were compared. The main methods are described below.

Three healthy plants of two ecotypes were taken as one replicate. The leaves were selected, washed with deionized water three times, dried with filter paper, then dried at $80{ }^{\circ} \mathrm{C}$ to a constant mass, and finally ground. Sample powder $(50 \mathrm{mg})$ was repeatedly extracted with ethanol three times and the supernatant was mixed. Then, activated carbon was added for decolorization and drying, followed by distilled water for constant volume. Finally, the soluble sugar was determined by anthrone colorimetry (Ebell 1969).

After weighing $500 \mathrm{mg}$ sample powder and adding $10 \mathrm{~cm}^{3} 3 \%$ sulfonated salicylic acid solution, centrifugation was performed at $12000 \mathrm{~g}$ for $10 \mathrm{~min}$, followed by filtration with filter paper. Finally, $2 \mathrm{~cm}^{3}$ supernatant was taken for determination of proline using the method of ninhydrin hydrate (Bates et al. 1973).

For chlorophyll determination, $0.5 \mathrm{~g}$ fresh samples were put into a mortar, ground with acetone, and the extract was used to determine the Chl content (Vernon 1960). The content of Chl $a$ and $b$ was measured using a spectrophotometer at $\mathrm{A}_{645}$ and $\mathrm{A}_{663}$ and calculated using the equations of Wintermans and De Mots (1965). Fresh samples $(1 \mathrm{~g})$ were cut into small fragments in a beaker and then macerated with $10 \mathrm{~cm}^{3} 0.1 \mathrm{M} \mathrm{HCl}$ and incubated at $32{ }^{\circ} \mathrm{C}$. Finally, the filtrate was taken for colorimetric determination of anthocyanin content (Wang et al. 2008a,b).

Total RNA extraction, library construction, and sequence analysis: Purslane was sequenced by Maine Health Technology Co. (Beijing, China). Total RNA was extracted from the roots, stems and leaves of two ecotypes of purslane (3 replicates in each group), and specific libraries were constructed after mRNA enrichment. The library was initially qualified with Qubit 3.0. After, the library was diluted to $1 \mathrm{mg} \mathrm{dm}^{-3}$, the insert size of the library was detected by Agilent 2100 . When the insert size met the expectation, the effective concentration of the library was accurately quantified by qPCR (the effective concentration of the library was more than $2 \mathrm{nM}$ ) to ensure the quality of the library. Then, the different libraries (downloaded on August 14, 2017) were HiSeq sequenced according to the effective concentration and the demand of target data. Assembled by Trinity (https://github.com/ trinityrnaseq/trinityrnaseq/wiki) software, clean reads were compared with the unigene sequence acquired by the assembly. Then the expressions of differentially expressed genes (DEGs) in different sample groups were analyzed according to their expressions (screened by DESeq, FDR $<0.01$ and fold change $>2$ as screening criteria), functional annotation and functional enrichment 
WU et al.

Table 1. Physiological indexes of red- and green-stem ecotypes of purslane. Different lowercase letters indicate statistically significant differences $(P<0.05)$.

\begin{tabular}{llllll}
\hline Purslane ecotypes & $\begin{array}{l}\text { Soluble sugar content } \\
{\left[\mathrm{mg} \mathrm{g}^{-1}(\mathrm{f} . \mathrm{m} .)\right]}\end{array}$ & $\begin{array}{l}\text { Free proline content }] \\
{\left[\mathrm{mg} \mathrm{g}^{-1}(\mathrm{f} . \mathrm{m} .)\right.}\end{array}$ & $\begin{array}{l}\text { Chlorophyll } a \text { content } \\
{\left[\mathrm{mg} \mathrm{g}^{-1}(\mathrm{f} . \mathrm{m} .)\right]}\end{array}$ & $\begin{array}{l}\text { Chlorophyll } b \text { content } \\
{\left[\mathrm{mg} \mathrm{g}^{-1}(\mathrm{f} . \mathrm{m} .)\right]}\end{array}$ & $\begin{array}{l}\text { Anthocyanin } \\
\text { content }\end{array}$ \\
\hline Red-stem & $14.95 \pm 0.295 \mathrm{a}$ & $4.17 \pm 0.244 \mathrm{a}$ & $0.66 \pm 0.001 \mathrm{a}$ & $0.61 \pm 0.004$ & $1.40 \pm 0.004 \mathrm{a}$ \\
Green-stem & $12.29 \pm 0.310 \mathrm{~b}$ & $3.27 \pm 0.240 \mathrm{~b}$ & $0.57 \pm 0.008 \mathrm{~b}$ & $0.60 \pm 0.003$ & $0.87 \pm 0.003 \mathrm{~b}$ \\
\hline
\end{tabular}

of differentially expressed genes (screened by $\log 2 \mathrm{FC}>$ 1.7 or $\log 2 \mathrm{FC}<-3.5$ as screening criteria) were analyzed. After the integration of down-regulated and up-regulated genes, gene ontology $(G O)$ classification (http://www. geneontology.org/) and functional annotation analysis of the $K E G G$ pathway (http://www.genome.jp/kegg/) were performed based on the $G O$ database.

RNA extraction and real-time qPCR analysis: Total RNA of purslane was extracted from leaves according to the instructions of the RNAprep Pure Plant kit (TIANGEN®, Beijing, China). The RNA concentration of each sample was measured by NanoDrop (Thermo Fisher Scientific, Massachusetts, USA). $1 \mu \mathrm{g}$ of total RNA was used for reverse transcription with HiScript ${ }^{\circledR} Q$ Select $R T$ SuperMix for qPCR. Real time PCR was performed using the above products and universal $S Y B R$ ( $q P C R$ Master Mix. The transcriptions of NAC, MYB, WRKY, and bZIP were detected by StepOnePlus (Applied Biosystems Inc., Shanghai, China) real-time PCR system. Triplicate replicates were performed in this study. The primer sequences of detected genes and internal reference gene primers (DN64753_c3_g4) are shown in Table 1 Suppl.

Statistical analysis: All data were submitted to the Bartlett test for homogeneity of variance, and then SPSS 20.0 (SPSS for Windows, Chicago, IL, USA) was used for $A N O V A$ analysis. An independent sample test was used to test the difference between the parameters of the two ecological types with a probability level of 0.05 , and the correlation between the parameters was determined using the least square method.

\section{Results}

To reveal underlying physiological differences in the two ecotypes of purslane, the physiological indicators of the red-stem and the green-stem purslanes were investigated. The soluble sugar content of the red-stem ecotype was $14.95 \mathrm{mg} \mathrm{g}^{-1}$, about $19.5 \%$ higher than that of the greenstem ecotype. Similar to the soluble sugar content, the content of proline, anthocyanins, and chlorophyll $a$ of the red-stem ecotype were all higher than those of the green-stem ecotype. However, there was no significant difference in the content of the chlorophyll $b$ between the two ecotypes of purslane (Table 1).

To explore the underlying molecular mechanism of physiological differences between two ecotypes of purslane, the transcriptome analysis of them was performed. A total of 29614 genes from the purslane transcription factor database were obtained. Compared with the green-stem purslane, a total of 1464 DEGs were identified including 923 up-regulated genes and 541 downregulated genes (Fig. 1).

The 1464 DEGs included $47 \mathrm{TF}$ families, and the distribution of these TFs was shown in Fig. 2 Most family members were involved in the regulation of drought stress, including AP2/ERF, WRKY, C2H2, NAC, bHLH, and MYB families. Among them, the MYB superfamily was the largest, including 15 (3.26\%) MYB and $28(6.09 \%)$ MYB-related members. The second largest family was the bHLH family with $31(6.74 \%)$ members, followed by the NAC (30, $6.52 \%), \mathrm{C} 2 \mathrm{H} 2(28,6.09 \%)$, WRKY (24,

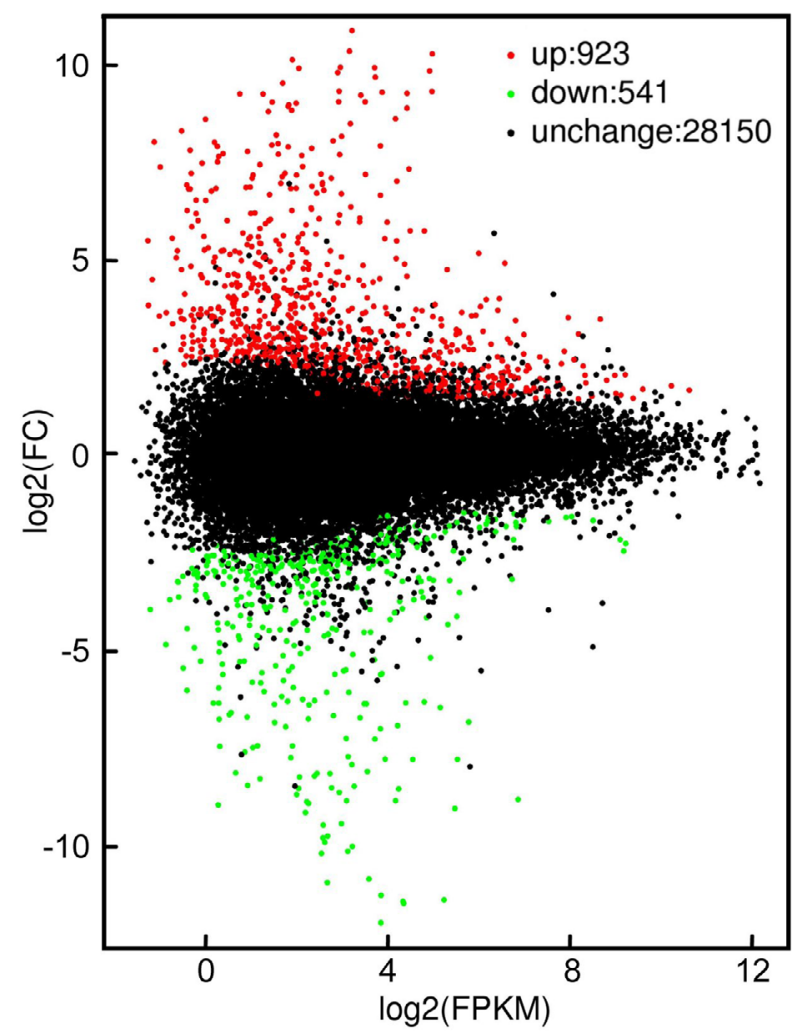

Fig. 1. Statistical map of a number of DEGs. The abscissa is $\log 2$ (FPKM), which is the logarithm of the mean expression of the two samples; the ordinate is $\log 2(\mathrm{FC})$, which is the logarithm of the multiple of gene expression difference between the two samples, which is used to measure the difference of expression (green-stem vs. red-stem ecotypes). The green dots represent down-regulated DEGs, the red dots represent up-regulated DEGs, and the black dots represent non-differentially expressed genes. 
5.22\%), and bZIP (16, $3.48 \%$ ) families (Fig. 1 Suppl.).

Many TF family members exhibited significant differences in their expressions in the two purslane ecotypes. The MYB superfamily was the TF family with the largest number of members (43 genes), and the second largest TF family was the bHLH family with 31 members, followed by the NAC (30), WRKY (24), bZIP (16), and AP2 (5) families (Fig. 3B). Among these TFs, the majority of DEGs of the NAC, bHLH, WRKY, AP2, MYB-related and MYB families were up-regulated in the red-stem ecotype compared with the green-stem ecotype, whereas most DEGs of the bZIP family were down-regulated (Fig. 3A). A large number of the identified TF genes have been investigated as key regulatory genes involved in plant abiotic stress responses. These results indicated that the TF families, such as NAC, bZIP, WRKY, AP2, bHLH, and MYB superfamily, might play important roles in the regulation of the red-stem ecotype tolerance to drought stress. In addition, the differential expressions of TFs in the same family demonstrated that different members may have distinct roles in plant response to drought stress and appear to exhibit differential biological functions.

To find identical amino acid sequences that some families expressed in purslane, the conserved domains of TFs were analyzed using the MEME program (http:// meme-suite.org). The identical amino acid sequences (PEFDGSLNVEDYLDWIHTVER) existed in the domains of the NAC, MYB-related, WRKY, and bHLH family; the identical amino acid sequences (IWWTNLQKKRVA) existed in the domains of AP2, MYB family (Fig. 4). The results suggested that TFs from different $\mathrm{TF}$ families may share the same regulation mechanism of the drought stress response.

After the integration of down-regulated and upregulated genes, $G O$ classification and functional annotation analysis of the $K E G G$ pathway were performed based on the $G O$ database (Tables 3 and 4 Suppl.). DEGs involved in biological functions were obtained by $G O$ classification. The results showed that they were involved in the regulation of the transduction of metabolic protein signals in plants, organelle activity, and intracellular and intercellular signal molecule transmission, the process of nucleic acid binding transcription factors, the activity of various proteins such as antioxidant enzymes and the mutual binding of proteins (Fig. 2 Suppl.). In terms of biological process, DEGs participate in DNA binding,

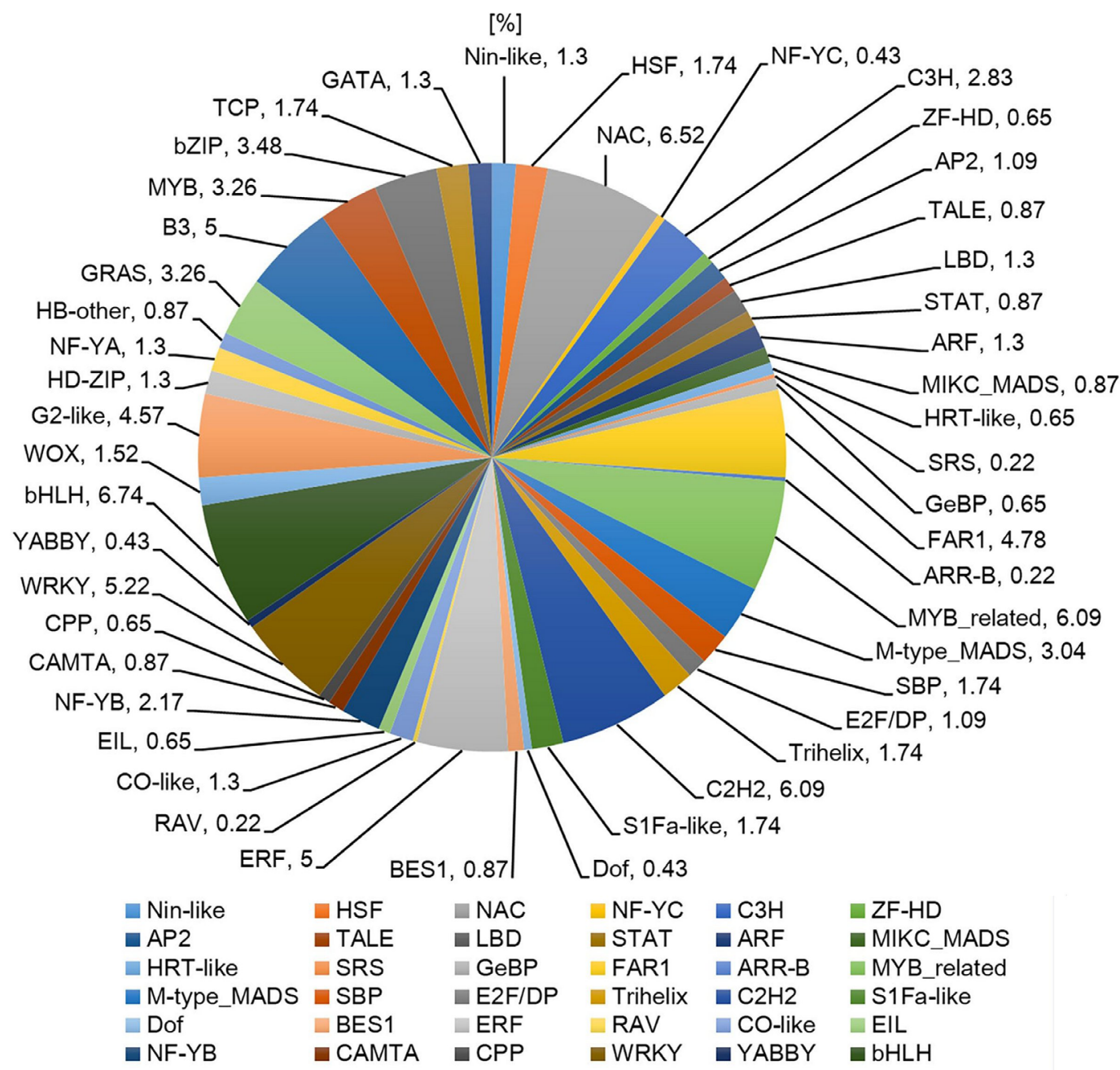

Fig. 2. Distribution of different TF families identified in the transcriptome analysis. TF families and their percentages are shown in the pie chart. 
protein phosphorylation, and so on, which can affect the replication and differentiation of cell, intercellular communication, and signal transmission. Analysis of cellular component showed that DEGs function in cell membrane composition. An analysis of molecular function showed that DEGs were involved in mediating the activity of transcription factors and transferases (Fig. 5).

$K E G G$ pathway analysis further interpreted gene function and enriched highly correlated signalling pathways. The results showed that the DEGs were mainly enriched in plant hormones signal transduction, from which it can be inferred that differential expressions of miRNAs were related to protein phosphatase, ABA receptors PYR-PYL-RCAR proteins and various abscisic acid (ABA) pathways (Fig. 3 Suppl.).

Eight typical genes were screened from DEGs of NAC, MYB, WRKY, and bZIP families by real-time qPCR, and the expression multiples of transcriptome analysis and $\mathrm{qPCR}$ validation of the same gene were shown in Table 5 Suppl. Although there were differences in their expression multiples, the results of qPCR validation

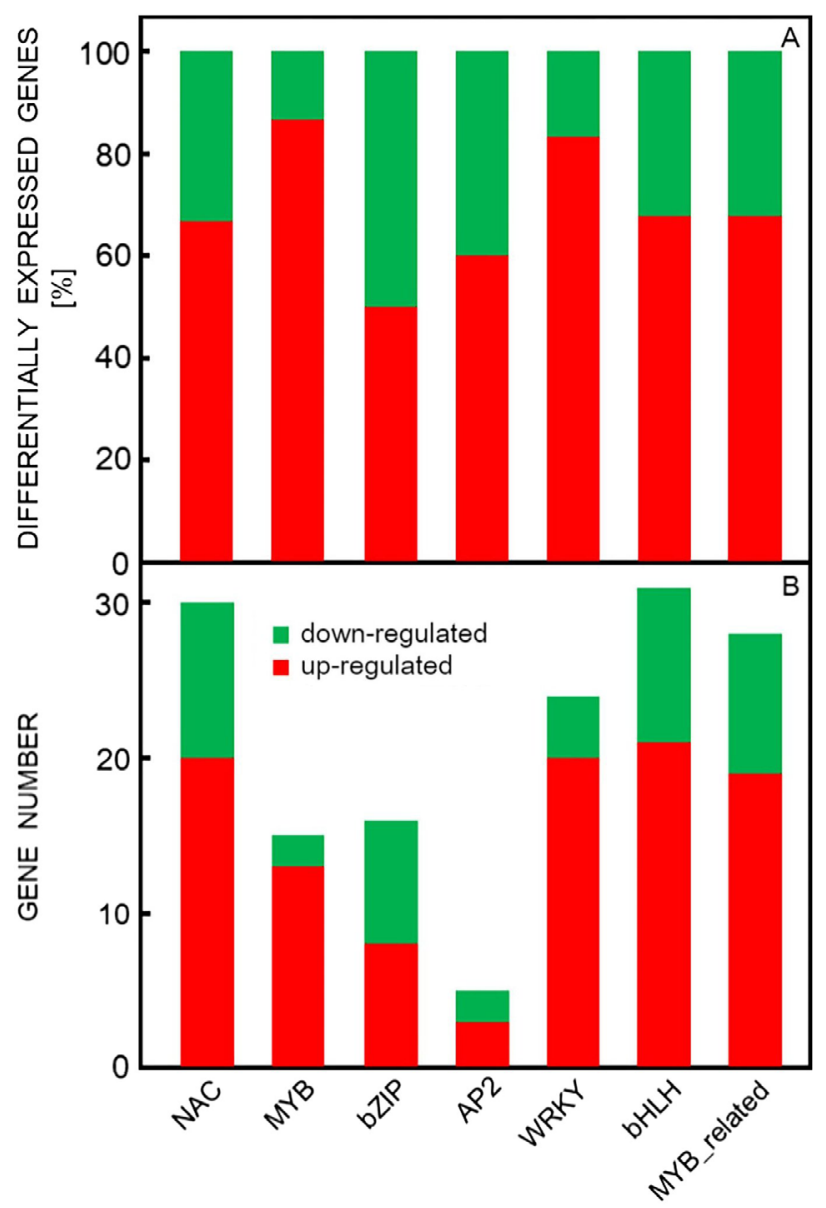

Fig. 3. Expression profiles of $\mathrm{TF}$ genes in the two ecotypes of purslane. $A$ - Percentages of up-regulated, and down-regulated $\mathrm{TF}$ genes in the red-stem ecotype compared with the green-stem ecotype. $B$ - The number of TF genes that were up-regulated and down-regulated in the red-stem ecotype compared with the green-stem ecotype. showed a significant correlation with transcriptome analysis $(P<0.05)$, which showed that the data obtained by transcriptional sequencing were reliable (Fig. 4 Suppl.).

\section{Discussion}

In the plant response mechanism to water deficit, morphophysiological responses mean that plants tend to enhance soil water uptake and osmotic adjustment by increasing the content of soluble sugars and proline under drought environment (Kumar et al. 2018). It has been shown that proline can alleviate the negative effects of plant water stress and so enhance plant tolerance to adverse environment. For example, the transgenic potato with increased expression of proline biosynthesis enzymes accumulated 1.25-times more proline under conditions of water shortage and grew better than the wild-type potato (Nie et al. 2018). Furthermore, some studies have shown that plants with high antioxidant capacity usually have high anthocyanin content, which makes the plants more resistant to various stresses, including salinity and drought (Sun et al. 2017, Tisarum et al. 2019). Also, the increase in Chl content could be explained as a compensatory mechanism to maintain photosynthetic activity under drought stress (Shobbar et al. 2012). In this paper, the soluble sugar, proline, anthocyanin, and chlorophyll content of the redstem ecotype were higher than those of the green-stem ecotype (Table 1), which was consistent with the previous report. Therefore, it was indicated that the physiological differences serve as indicators in evaluating different drought resistance of the red-stem ecotype and the greenstem ecotype, moreover, anthocyanins and chlorophylls also play a significant role in the adaptation mechanism of Portulaca oleracea (Gonzáles-Vilagra et al. 2017, Saheri et al. 2020).

With the rapid development of molecular biology, many physiological and biochemical characteristics can be further explored at the molecular level. Currently, it has been found that plants can effectively cope with drought stress through a series of genes such as $A P 2 / E R F$, WRKY, $b Z I P, N A C$ and $M Y B$ related genes, and TFs play a major role in plant response strategies to cope with adverse conditions (Yang et al. 2012, Zhang et al. 2012, Jan et al. 2013, Wang et al. 2013, Shingote et al. 2015, Du et al. 2018, Goel et al. 2019). Overexpression or disruption of the OsNAC5 gene may affect soluble sugar and proline content in plants (Song et al. 2011). Co-expression of MdMYB308L and $M d b H L H 33$ enhances the production of anthocyanin in apple, which is conducive to enhancing its resistance (An et al. 2019). The BdWRKY36 overexpression causes lesser ion leakage and reactive oxygen species (ROS) accumulation, but higher content of chlorophyll, relative water content, and activities of antioxidant enzymes under drought conditions (Sun et al. 2015). Arabidopsis transgenic plants overexpressing TabZIP show enhanced tolerance to salt, drought, heat, and oxidative stresses, and overexpression of TaMYB33 increases salt and drought tolerance (Qin et al. 2012). Overexpression of StDREB2 (AP2) increases resistance to drought in cotton and the 
overexpressions of StERF147, StERF169, StERF120, and StERF110 improve heat tolerance in potato (Agarwal et al. 2019, El-Esawi and Alayafi 2019). In this study, we identified the drought-tolerant TF genes in two purslane ecotypes by using comparative transcriptome analysis.
Here, among the total of 29614 genes from the purslane transcription factor database, 1464 DEGs were identified between two purslane ecotypes (Fig. 1). Meanwhile, 1464 $\mathrm{TF}$ genes were identified in the transcriptome and divided into $47 \mathrm{TF}$ families (Fig. 2), which were differentially

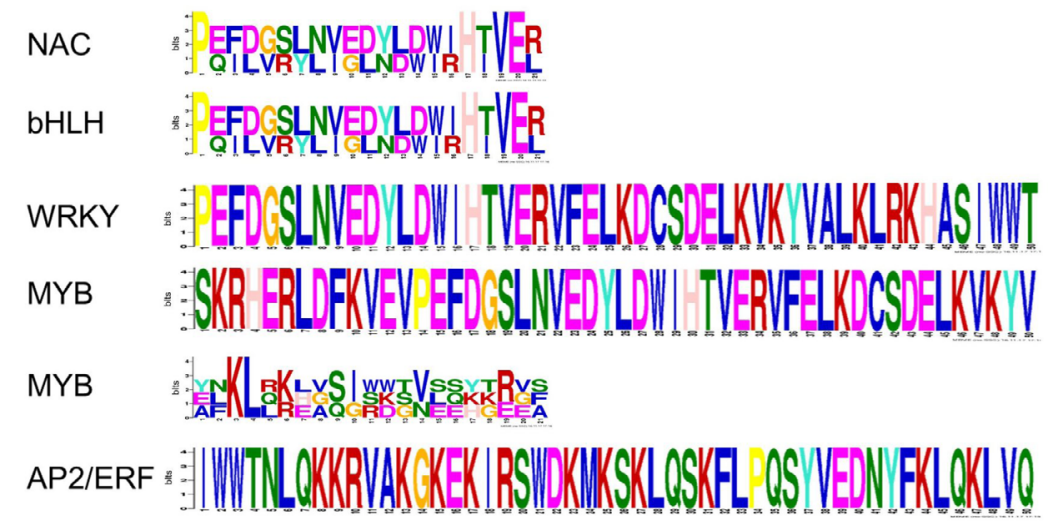

Fig. 4. Sequence logos of TFs domains in purslane. The total height of the stack indicates the information content of that position in the motif. The height of residues within the stack indicates the probability of each residue at that position.

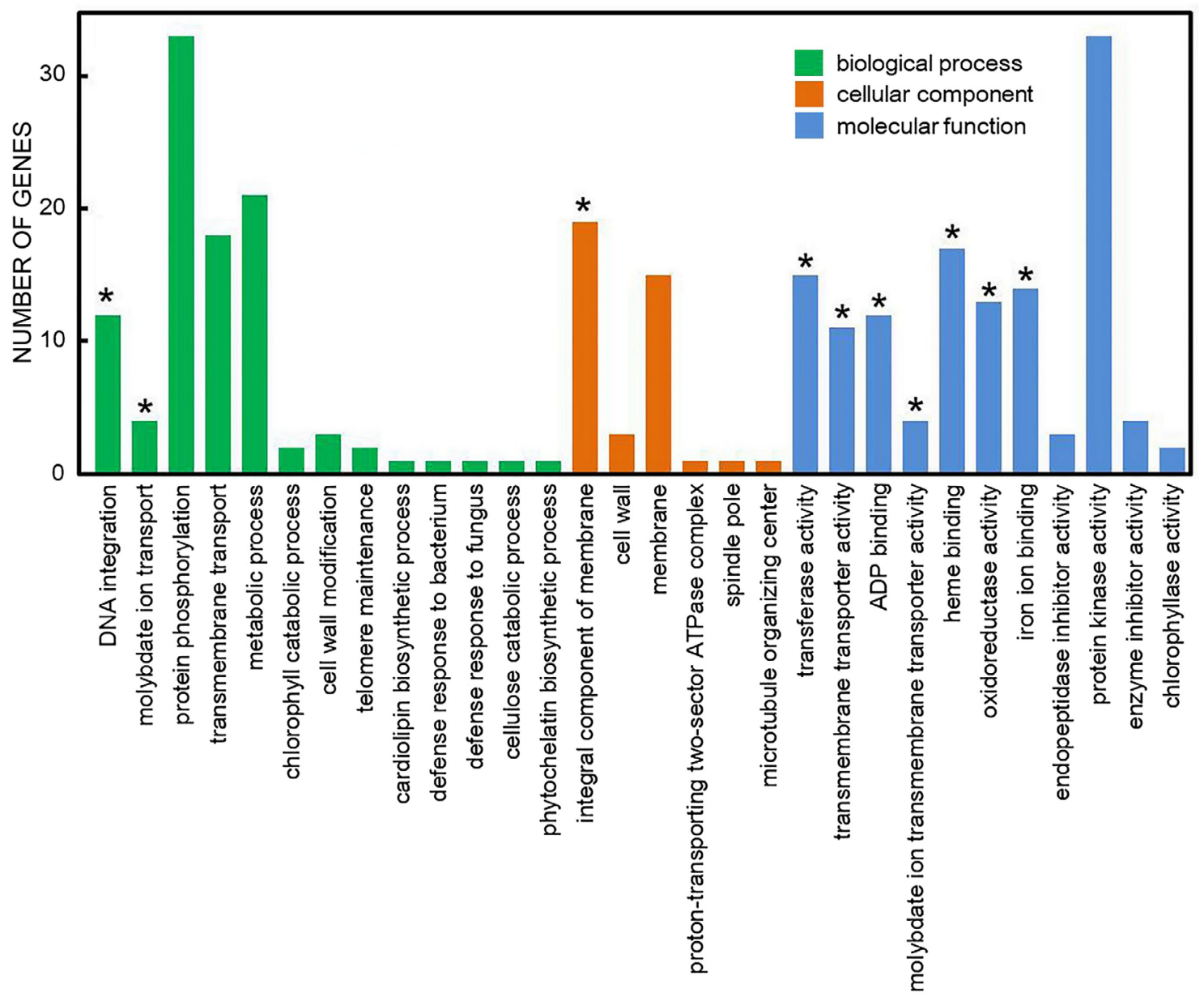

Fig. 5. Go enrichment histogram (all ranked in the top 30). The horizontal coordinate is the enriched GO term, and the vertical coordinate is the number of differential genes in the term. Different colours are used to distinguish biological processes, cellular components and molecular functions. * indicates that the enrichment trend of differentially expressed genes is different from that of all genes. It can be focused on whether this function is related to the difference. 
expressed in the two purslane ecotypes. In other words, a group of TF genes under drought stress had significant differences in the expression in red- and green-stem purslane. Therefore, it was concluded that DEGs do exist between two purslane ecotypes, moreover, these $\mathrm{TF}$ families were involved in plant responses to drought stress, including AP2/ERF, WRKY, bZIP, NAC, and MYB families, and might play a key role in the adaptation of purslane to drought.

To identify the key genes involved in the formation of differences between two ecotypes of purslane, the conserved domains of drought tolerance regulatory families such as the bHLH family and NAC family were analyzed by comparative analysis and it was found that the same amino acid sequences existed among different families (Fig. 4), and the same amino acid sequences are likely to end up as the same functional protein (Wu et al. 2015). Combined with the results of physiological indicators, it was inferred that these gene families responded to the drought by producing similar functional proteins. Generally speaking, under the condition of water shortage, plants are mainly regulated by the comprehensive coordination of the ABA regulation system (Baldoni et al. 2015). Meanwhile, Butt et al. (2017) found that GaMYB85 positively regulates ABA-induced stomatal density, stomatal size, and stomatal opening rates.

Compared with untransformed tobacco, TaWRKY1expressing plants can improve its drought tolerance by changing a series of osmotic stress defense processes related to abscisic acid dependent pathways, such as osmolyte accumulation, stomata movement characterization, and plant water retention behavior (Ding et al. 2016). Moreover, it was also found that accumulation of endogenous ABA seemed to be involved in water stress-induced accumulation of proline in leaves of drought-tolerant wheat (Saeedipour 2013). In addition, there are some WRKY proteins in many plants that have different regulatory effects on ABA and induction of plant tolerance, mainly drought. For example, OsWRKY45-1 and $O s W R K Y 45-2$ genes in rice play negative and positive roles in the ABA signalling pathway, respectively (Tao et al. 2011). Jiang et al. (2012) also suggested that the activated expression of AtWRKY57 improved the drought tolerance of Arabidopsis by elevating ABA content. Furthermore, we can further infer that in two different ecotypes of purslane, the differential genes of the TFs family probably regulate physiological indicators such as proline content through the ABA signalling pathway and further participate in the response of purslane to drought stress (Saeedipour 2013). It can be found from Fig. 5 that in the enrichment trend of DEGs, there is a significant difference in signalling, indicating that this function is related to different ecotypes of purslane. In this paper, $G O$ enrichment function annotation was carried out on the DEGs of two different ecotypes of purslane and sorted out the expression of up-regulated and down-regulated genes (Tables 3 and 4 Suppl.), so it was found that the genes were mainly related to ABA signalling pathway, which was consistent with the inferred results.

To further confirm the effect of the TF family on the response of purslane to drought stress, eight genes of the NAC, MYB, WRKY, and bZIP family in red-stem and green-stem purslane were selected for real-time qPCR. The results showed that the expression trend was basically the same as found in transcriptome analysis, and there was a significant correlation between the expression trend and sequencing trend (Fig. 4 Suppl.). So it was speculated that these transcription factors might be involved in the adaptation to the drought environment through mediating the content of soluble sugar, proline, and anthocyanins, and the related metabolic processes. However, the mechanism underlying the role of these predicted transcription factors in purslane under drought stress needs to be further explored. By comparing and analyzing the transcriptomes of the red-stem purslane and the green-stem purslane under drought stress, the difference of gene expression is helpful to deepen the understanding of TFs of purslane involved in the relevant response strategies under drought stress, and to provide a reference basis for the next study of gene function, and to provide a theoretical basis for the study of new stress-resistant cultivars by genetic engineering.

\section{References}

Agarwal, P., Baranwal, V.K., Khurana, P.: Genome-wide analysis of bZIP transcription factors in wheat and functional characterization of a TabZIP under abiotic stress. - Sci. Rep. 9: 4608, 2019.

Alam, M.A., Juraimi, A.S., Rafii, M.Y., Hamid, A.A., Aslani, F.: Screening of purslane (Portulaca oleracea L.) accessions for high salt tolerance. - Sci. World J. 2014: 627916, 2014a.

Alam, M.A., Juraimi, A.S., Rafii, M.Y., Hamid, A.A., Aslani, F.: Collection and identification of different purslane (Portulaca oleracea L.) accessions available in Western Peninsular Malaysia. - Life Sci. J. 11: 431-437, 2014b.

Alam, M.A., Juraimi, A.S., Rafii, M.Y., Hamid, A.A., Aslani, F., Hakim, M.A.: Salinity-induced changes in the morphology and major mineral nutrient composition of purslane (Portulaca oleracea L.) accessions. - Biol. Res. 49: 1-19, 2016.

Ammar, M.H., Anwar, F., El-Harty, E.H., Migdadi, H.M., AbdelKhalik, S.M., Al-Faifi, S.A., Farooq, M., Alghamdi, S.S.: Physiological and yield responses of faba bean (Vicia faba L.) to drought stress in managed and open field environments. - J. Agron. Crop Sci. 201: 280-287, 2014.

An, J.P., Wang, X.F., Zhang, X.W., Xu, H.F., Bi, S.Q., You, C.X., Hao, Y.J.: An apple MYB transcription factor regulates cold tolerance and anthocyanin accumulation and undergoes MIEL1-mediated degradation. - Plant Biotechnol. J. 18: 337 353,2019

Baldoni, E., Genga, A., Cominelli, E.: Plant MYB transcription factors: their role in drought response mechanisms. - Int. J. mol. Sci. 16: 15811-15851, 2015.

Bates, L.S., Waldren, R.P., Teare I.D.: Rapid determination of free proline for water-stress studies. - Plant Soil 39: 205-207, 1973.

Butt, H.I., Yang, Z., Gong, Q., Chen, E., Wang, X., Zhao, G., Ge, X., Zhang, X., Li, F.: GaMYB85, an R2R3 MYB gene, in transgenic Arabidopsis plays an important role in drought tolerance. - BMC Plant Biol. 17: 142, 2017.

Conesa, M.R., De la Rosa, J.M., Domingo, R., Bañon, S., Pérez-Pastor, A.: Changes induced by water stress on water relations, stomatal behaviour and morphology of table grapes 
(cv. Crimson Seedless) grown in pots. - Sci. Hort. 202: 9-16, 2016.

Ding, W., Fang, W., Shi, S., Zhao, Y., Li, X., Xiao, K.: Wheat WRKY type transcription factor gene TaWRKY1 is essential in mediating drought tolerance associated with an ABAdependent pathway. - Plant Mol. Biol. Rep. 34: 11111126, 2016.

Du, Y.T., Zhao, M.J., Wang, C.T., Gao, Y., Wang, Y.X., Liu, Y.W., Chen, M., Chen, J., Zhou, Y.B., Xu, Z.S., Ma, Y.Z.: Identification and characterization of GmMYB118 responses to drought and salt stress. - BMC Plant Biol. 18: 320, 2018.

Ebell, L.F.: Variation in total soluble sugars of conifer tissues with method of analysis. - Phytochemistry 8: 227-233, 1969.

El-Esawi, M.A., Alayafi, A.A.: Overexpression of StDREB2 transcription factor enhances drought stress tolerance in cotton (Gossypium barbadense L.). - Genes 10: 142, 2019.

Goel, P., Bhuria, M., Sinha, R., Sharma, T.R., Singh, A.K.: Promising transcription factors for salt and drought tolerance in plants. - In: Singh, S.P., Upadhyay, S.K., Pandey, A., Kumar, S. (ed): Molecular approaches in plant biology and environmental challenges. Pp. 7-50. Springer, Singapore 2019.

González-Villagra, J., Kurepin, L.V., Reyes-Díaz, M.M.: Evaluating the involvement and interaction of abscisic acid and miRNA156 in the induction of anthocyanin biosynthesis in drought-stressed plants. - Planta 246: 299-312, 2017.

Haworth, M., Elliott-Kingston, C., McElwain, J.C.: Coordination of physiological and morphological responses of stomata to elevated $\left[\mathrm{CO}^{2}\right]$ in vascular plants. - Oecologia 171: 71-82, 2013.

Jan, A., Maruyama, K., Todaka, D., Kidokoro, S., Abo, M., Yoshimura, E., Shinozaki, K., Nakashima, K., YamaguchiShinozaki, K.: OsTZF1, a CCCH-tandem zinc finger protein, confers delayed senescence and stress tolerance in rice by regulating stress-related genes. - Plant Physiol. 161: 12021216, 2013.

Jiang, Y., Qiu, Y., Hu, Y., Yu, D.: Heterologous expression of AtWRKY57 confers drought tolerance in Oryza sativa. - Front. Plant Sci. 7: 145, 2016.

Jiang, Y.J., Liang, G., Yu, D.Q.: Activated expression of WRKY57 confers drought tolerance in Arabidopsis. - Mol. Plants 5: 1375-1388, 2012.

Kafi, M., Rahimi, Z.: Effect of salinity and silicon on root characteristics, growth, water status, proline content and ion accumulation of purslane (Portulaca oleracea L.). - Soil Sci. Plant Nutr. 57: 341-347, 2011.

Kumar, S., Sachdeva, S., Bhat, K.V., Vats, S.: Plant responses to drought stress: physiological, biochemical and molecular basis. - In: Vats, S. (ed.): Biotic and abiotic stress tolerance in plants. Pp. 1-25. Springer, Singapore 2018.

Macková, H., Hronková, M., Dobrá, J., Turečková, V., Novák, O., Lubovská, Z., Motyka, V., Haisel, D., Hájek, T., Prášil, I.T., Gaudinová, A., Štorchová, H., Ge, E., Werner, T., Schmülling, T., Vaňková, R.: Enhanced drought and heat stress tolerance of tobacco plants with ectopically enhanced cytokinin oxidase/dehydrogenase gene expression. - J. exp. Bot. 64: 2805-2815, 2013.

Nie, L.Z., Yu, X.X., Ma, Y.H., Fang, Y.Y., Li, L.M., Yu, Z.: Enhanced drought and osmotic stress tolerance in transgenic potato plants expressing $A t C D P K_{l}$, a calcium-dependent protein kinase. - Russ. J. Plant Physiol. 65: 865-873, 2018.

Pennisi, E.: How plants learned to breathe. - Science 355: 11101111, 2017.

Phillips, G.N.,Jr., Sussman, M.R.: Plant hydraulics and agrichemical genomics. - Science 366: 416-417, 2019.

Qin, Y., Wang, M., Tian, Y., He, W., Han, L., Xia, G.: Over- expression of TaMYB33 encoding a novel wheat MYB transcription factor increases salt and drought tolerance in Arabidopsis. - Mol. biol. Rep. 39: 7183-7192, 2012.

Saeedipour, S.: Relationship of grain yield, ABA and proline accumulation in tolerant and sensitive wheat cultivars as affected by water stress. - Proc. nat. Acad. Sci., India Sect. B Biol. Sci. 83: 311-315, 2013.

Saheri, F., Barzin, G., Pishkar, L., Akbar Boojar, M.M., Babaeekhou, L.: Foliar spray of salicylic acid induces physiological and biochemical changes in purslane (Portulaca oleracea L.) under drought stress. - Biologia 75: 2189-2200, 2020.

Shingote, P.R., Kawar, P.G., Pagariya, M.C., Kuhikar, R.S., Thorat, A.S., Babu, K.H.: SoMYB18, a sugarcane MYB transcription factor improves salt and dehydration tolerance in tobacco. - Acta physiol. Plant. 37: 217, 2015.

Shobbar, M.S., Azhari, O., Shobbar, Z.S., Niknam, V., Pessarakli, M., Ebrahimzadeh, H.: Comparative analysis of some physiological responses of rice seedlings to cold, salt, and drought stresses. - J. Plant Nutr. 35: 1037-1052, 2012.

Simopoulos, A.P.: The importance of the omega-6/omega-3 fatty acid ratio in cardiovascular disease and other chronic diseases. - Exp. Biol. Medicine 233: 674-688, 2008.

Song, S.Y., Chen, Y., Chen, J., Dai, X.Y., Zhang, W.H.: Physiological mechanisms underlying OsNAC5-dependent tolerance of rice plants to abiotic stress. - Planta 234: 331345,2011

Sun, J., Hu, W., Zhou, R., Wang, L., Wang, X., Wang, Q., Feng, Z., Li, Y., Qiu, D., He, G., Yang, G.: The Brachypodium distachyon BdWRKY36 gene confers tolerance to drought stress in transgenic tobacco plants. - Plant Cell Rep. 34: 2335, 2015.

Sun, M., Feng, X.X., Gao, J.J., Peng, R.H., Yao, Q.H., Wang, L.J.: VvMYBA6 in the promotion of anthocyanin biosynthesis and salt tolerance in transgenic Arabidopsis. - Plant Biotechnol. Rep. 11: 299-314, 2017.

Tao, Z., Liu, H.B., Li, X.H., Xiao, J.H., Wang, S.P.: OsWRKY45 alleles play different roles in abscisic acid signalling and salt stress tolerance but similar roles in drought and cold tolerance in rice. - J. exp. Bot. 62: 4863-4874, 2011.

Tisarum, R., Theerawitaya, C., Samphumphuang, T., Phisalaphong, M., Singh, H.P., Cha-Um, S.: Promoting water deficit tolerance and anthocyanin fortification in pigmented rice cultivar (Oryza sativa L. subsp. indica) using arbuscular mycorrhizal fungi inoculation. - Physiol. mol. Biol. Plants 25: 821-835, 2019.

Uddin, M.K., Juraimi, A.S., Ali, M.E., Ismail, M.R.: Evaluation of antioxidant properties and mineral composition of purslane (Portulaca oleracea L.) at different growth stages. - Int. J. mol. Sci. 8: 10257-10267, 2012.

Vernon, L.P.: Spectrophotometric determination of chlorophylls and pheophytins in plant extracts. - Anal. Chem. 32: 1144$1150,1960$.

Wang, F., Deng, J.H., Tan, X.H., Zhang, L.H., Zhong, H., Li, Q.M.: Research progress on anthocyanins and copigmentation. - Food Sci. 29: 472-476, 2008a.

Wang, Q., Li, X., Wang, L., Sun, F., Shen, X.: Dynamic changes of anthocyanin and the relevant biosynthesis enzymes in Padus virginiana 'Schubert' leaves. - Sci. silvae sin. 44: 4549, 2008b.

Wang, R.K., Cao, Z.H., Hao, Y.J.: Overexpression of a R2R3 MYB gene MdSIMYB1 increases tolerance to multiple stresses in transgenic tobacco and apples. - Physiol. Plant. 150: 76-87, 2013.

Wintermans, J.F., De Mots, A.: Spectrophotometric characteristics of chlorophylls $a$ and $b$ and their pheophytins in ethanol. - 
Biochim. biophys. Acta. 109: 448-453, 1965.

Wu, Z.J., Li, X.H., Liu, Z.W., Li, H., Wang, Y.X., Zhuang, J.: Transcriptome-based discovery of AP2/ERF transcription factors related to temperature stress in tea plant (Camellia sinensis). - Funct. integr. Genomics 15: 741-752, 2015.

Yang, A., Dai, X.Y., Zhang, W.H.: A R2R3-type MYB gene, $O S M Y B 2$, is involved in salt, cold, and dehydration tolerance in rice. - J. exp. Bot. 63: 2541-2556, 2012.
Yazici, I., Türkan I., Sekmen, A.H., Demiral, T.: Salinity tolerance of purslane (Portulaca oleracea L.) is achieved by enhanced antioxidative system, lower level of lipid peroxidation and proline accumulation. - Environ. exp. Bot. 61: 49-57, 2007.

Zhang, L., Zhao, G., Xia, C., Jia, J., Liu, X., Kong, X.: A wheat R2R3-MYB gene, TaMYB30-B, improves drought stress tolerance in transgenic Arabidopsis - J. exp. Bot. 63: 5873$5885,2012$. 